\title{
Processing Maps for the Hot Forming of Polycrystalline Metallic Materials Using the Garofalo Equation
}

\begin{abstract}
IGNACIO RIEIRO and OSCAR A. RUANO
Processing maps have been built for $>30$ years to predict the stable conditions for metal forming at high temperatures and strain rates. These maps, which also include efficiency conditions, are based on the stability criterion of Ziegler-Prasad (Z-P) within the framework of the dynamical materials model (DMM). In the present work, this criterion is drastically modified by introducing a Garofalo equation in the expression of the dissipative co-content, $J$, maintaining its definition in the framework of the DMM. This modification applies to its derivative with respect to the strain rate and also to the definition of efficiency, $\eta$. This new parameter developed in this work, named rho, $\rho$, is different from the stability parameter, xi, $\xi$, of the Z-P criterion since it is based on different constitutive equations. Rho is based on a Garofalo equation and xi on a power law equation. Both are obtained from experimental data and give rise to different stability maps that can be applied to any polycrystalline metallic material. In this work, a well-documented Ti-10V-4.5Fe alloy has been chosen to contrast the differences and similarities between the two methods of characterizing the stability conditions. Special emphasis is given to the predictions for the optimum forming temperatures.
\end{abstract}

https://doi.org/10.1007/s11661-020-05959-y

(C) The Minerals, Metals \& Materials Society and ASM International 2020

\section{INTRODUCTION}

FOR more than 30 years several models have been developed for the determination of the most stable areas, or zones of stability, in the process of hot working of metallic materials. There has also been interest in analyzing the zones of greater thermodynamic efficiency of these processes, something necessary for the optimization and research on new materials. Consequently, these models have been used to calculate the values of the efficiency of the process of plastic flow of hot working of polycrystalline metallic materials. These calculations of efficiency and stability of the mentioned processes can be represented in maps as the temperature versus true strain rate (plane $T, \dot{\varepsilon}$ ) of the processes. This gives rise to efficiency and stability maps that, joined, constitute the so-called processing maps.

The theoretical frame of reference for the study of stability of dynamical systems starts with the theoretical works of Lyapunov in 1892, first through his $\mathrm{PhD}$

IGNACIO RIEIRO is with the Department of Mathematics, Universidad de Castilla-La Mancha, Av. Carlos III s/n, 45071 Toledo, Spain. OSCAR A. RUANO is with the Department of Physical Metallurgy, CENIM-CSIC, Av. Gregorio del Amo 8, 28040 Madrid, Spain. Contact e-mail: ruano@cenim.csic.es.

Manuscript submitted November 21, 2019.

Article published online September 2, 2020 thesis ${ }^{[1]}$ and later through various works, ${ }^{[2,3]}$ and in the theoretical work of Ziegler. ${ }^{[4]}$ This last work helped to approximate the theoretical framework to a more realistic referential frame than that of the general works of Lyapunov that were more theoretical. On the other hand, Ziegler uses the concepts of thermodynamics of irreversible processes and also thermomechanics and mechanics of continuous media.

The criteria of Lyapunov and those of Ziegler predict different conditions of temperature and strain rate for the stability of materials but both are valid to study the hot-working behavior of materials. In the present work, the Lyapunov criteria are not considered since this was done in a previous publication by Rieiro et al. ${ }^{[5]}$ In the present work, only the stability criterion of $\mathrm{Ziegler}^{[4]}$ is analyzed, which is related to plastic flow at large strains. Ziegler uses the mechanics of the continuum for the case of large plastic flow and especially the thermodynamics of irreversible processes, considering extreme principles.

Later, and using the previously mentioned frameworks, the dynamic materials model, DMM, was introduced, attributed to Prasad. ${ }^{[6]}$ This model, over the years, has suffered criticism, modifications, extensions and reviews by very different researchers, but it resists as a model to evaluate the stability (among other objectives of the model). Because of their importance in clarifying DMM, the works of Gegel, Malas et al., should be mentioned. ${ }^{[7,8]}$ 
The DMM is based on the previously referenced theoretical frameworks, ${ }^{[6-8]}$ and a line of stability criteria applied to specific metallic materials under hot-forming conditions is set. The DMM is the synthesis and confluence of the dynamic theory of material systems based on concepts of energy combined with thermodynamics: the thermodynamics of irreversible processes and the theory of the stability of dynamic systems in the mechanics of continuous medium applied to large plastic flow.

The scope of the DMM is much broader than simply obtaining forming maps that are conceived as a synthesis of stability maps and efficiency maps. Over the years, this model has by far been the most used in the literature to draw processing maps in the field of plastic flow for the hot forming of polycrystalline metallic materials. The theoretical framework of Ziegler or that of Lyapunov can be applied, although with different results, to the concepts of DMM.

Two contributions of Prasad, Kumar and others should be clearly differentiated. The first, and most important, is the application of the concepts of stability of Ziegler (and of Lyapunov) to the field of hot working of materials. The second contribution is the construction of stability maps where Prasad and Kumar identify the parameters describing the plastic deformation, within the DMM, with the stability equations of Ziegler. They use an equation that relates temperature, $T$, stresses, $\sigma$, and strain rates, $\dot{\varepsilon}$, which gives rise to the analysis of constitutive equations. This last contribution led to numerous contradictions and critiques since these authors propose a power law as the constitutive equation. More specifically, they propose a power law for each of the ranges of strain rate and temperature that are divided the experimental data. In some cases, the authors use constitutive equations created ad hoc from polynomial expressions dependent on temperature, stress and strain ${ }^{[9]}$ although the theoretical basis is the power law within the framework of the DMM. As pointed out by Murty et al., 1999-2005, in several of their works, ${ }^{[10-13]}$ when a power law is used, the strain rate sensitivity, $\mathrm{m}$, and other parameters, turns out to be constant.

The Garofalo equation is admitted as a constitutive equation that adjusts large ranges of variables for hot forming of polycrystalline metallic materials. ${ }^{[14,15]} \mathrm{On}$ the other hand, it is important that the results of the application of a constitutive equation have behaviors that coincide with those that can be obtained from microstructural or atomistic models. We considered that it was necessary that the framework for assessing the stability conditions of the hot-working process be based on the work of Raj. ${ }^{[16,17]}$ and Raghunhat et al. ${ }^{[18]} \mathrm{Raj}$ has modeled atomistic mechanisms that are responsible for the microstructural damage caused by the hot-working process and has characterized the temperature and deformation rate conditions where this damage occurs. Raghunhat et al. ${ }^{[18]}$ have interpreted these conditions in the form of processing maps, as that given in Figure 1, in terms of ductile fracture due to voids, occurring at high strain rates and low temperatures, and the formation of wedge cracking at grain boundary triple

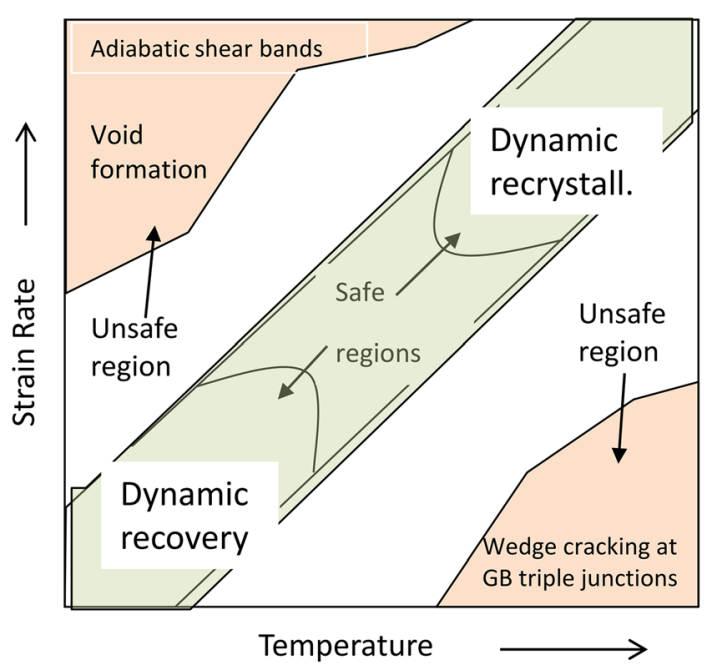

Fig. 1-Interpretation of the safe and unsafe regions according to the work of Raj ${ }^{[16,17]}$ and Raghunhat et al..$^{[18]}$

junctions, occurring at low strain rates and high temperatures. The area limited by the boundary conditions for these two damage processes is referred to "as safe for hot forming" while the upper limit of strain rate is set by the formation of "adiabatic shear bands."

In the safe or stable regime, two processes occur. ${ }^{[16,17]}$ One called dynamic recovery occurs at low strain rates and low temperatures, and the other, called dynamic recrystallization, occurs at high temperatures and high strain rates. The different domains shown by the power dissipation maps can be identified based on the corresponding efficiency variations of the microstructural characteristics, as indicated by Genesan et al. ${ }^{[19]}$ Changes in microstructures, modification of treatment and other processes can modify this general theoretical framework, but it is a good reference to consider.

In the present work a criterion is built, in the framework of DMM, based on the Ziegler-Prasad theory, using the Garofalo equation as a constitutive equation. This equation replaces the power law equation used by Prasad et al. ${ }^{\left[{ }^{6-8]}\right.}$ It should be noted that the criterion proposed in this work is different from the Z-P criterion and also different from the xi, $\xi$, stability parameter of Prasad. ${ }^{[20,21]}$

The Garofalo equation, due to its wide range of applicability of the thermomechanical variables, will avoid the polynomials built "ad hoc" and also the use of a power law. The Garofalo equation is widely used and is accepted as the best representative algebraic expression of the behavior of the thermomechanical variables for plastic flow for large strains in the hot-forming process as discussed in the works of Wang et al., ${ }^{[22]}$ and Wan et al., ${ }^{[23]}$ Section III describes the introduction of the Garofalo equation in the fundamental equations of the Ziegler-Prasad theory in the framework of DMM. This step has been put aside over the years because of the mathematical complexity of the problem, but it is recognized that the Garofalo equation is the correct one to correlate the data. We have solved the mathematical 
problem in some aspects analytically and in others through the application of numerical calculation methods. It was necessary to use simple variable changes, the chain rule for derivation of compound functions and the Leibniz rule for differentiation under the integral sign. This is a paramount work, and the details of the steps to reach these equations are given in the Appendix.

An investigation of the hot-working behavior of Ti-10V-4.5Fe-1.5Al alloy by Balasubrahmanyam and Prasad $^{[24]}$ (B-P) was selected because data of $\{T, \sigma, \dot{\varepsilon}\}$ are given explicitly in the form of tables. This avoids extracting data from graphs, which always has errors not allowing the elaboration of the procedures described in this work and a correct comparison with other interpretations procedures. The same procedure can be followed to study the hot-working behavior of any material that deforms plastically just by considering the corresponding values of the Garofalo parameters. It can also be applied to compression, torsion or tensile tests.

The objective of the present work, therefore, is linked to two secondary objectives. The first is to achieve theoretical improvements, within the framework of the DMM, by substituting the power law equation by a Garofalo equation, capable of predicting wide ranges of the thermomechanical variables for hot forming. The second objective is to be able to develop a methodology that reduces the possible numerical errors that can occur in the processes of generation of isolines (contour curves) in the stability maps. Therefore, the maps generated by Prasad et al. using the xi parameter are replaced by those generated using a new stability rho, $\rho$, parameter, but both under the general Ziegler-Prasad criterion.

\section{GENERAL THEORETICAL FRAMEWORK}

Ziegler $^{[4]}$ developed a theoretical model for plastic flow, based on the physical laws of thermodynamics of irreversible processes. Ziegler arrives at a stability condition for the dissipation function in relation to the variability of the thermodynamic function of the system as follows:

$$
\frac{\partial D}{\partial R}>\frac{D}{R}
$$

with $D$ being the dissipation function of the system and $R$ a distance in velocity space. ${ }^{[4]}$

This expression is used in general works in the framework of DMM, as for instance in the works of Lu et al. ${ }^{[9]}$ and Sahoo et al. ${ }^{[25]}$ and is transformed into $(R$ $=\dot{\varepsilon})$ :

$$
\frac{\partial D}{\partial \dot{\varepsilon}}>\frac{D}{\dot{\varepsilon}}
$$

From Eq. [2] it is deduced that the irreversible force that directs the dynamics of the system increases with the deformation rate. In addition, the law of increase must be logarithmic as it occurs in many physical laws:

$$
\frac{\partial \operatorname{Ln}(D)}{\partial \operatorname{Ln}(\dot{\varepsilon})}>1
$$

The conditions of stable plastic flow, or the stability criterion of Ziegler corresponding to the dynamic evolution of physical systems (Eq. [2]), are applied by Prasad et al. to any material by defining, in the DMM framework, the dissipative energy content $D$. At the end of this process of applying the DMM to Eq. [2], the criterion for stability, xi, known as the stability criterion of Ziegler-Prasad, is reached.

Kumar and Prasad, and their co workers, ${ }^{[6,26-31]}$ developed a practical criterion to analyze the stability of the various regions of strain rate and temperature during the hot-working process. They started from the dissipated power, $P(i)$, that in the framework of DMM is fractioned in two parts. One is $J$, whose original energy is a potential energy, and the other is $G$, whose original energy is a kinetic energy. Under the DMM they are named $J$, the dissipator co-content, and $G$, the dissipator content, according to the relation:

$$
P=G+J=\sigma \cdot \dot{\varepsilon}=\int_{0}^{\dot{\varepsilon}} \sigma d \dot{\varepsilon}+\int_{0}^{\sigma} \dot{\varepsilon} d \sigma
$$

with

$$
J=\int_{0}^{\sigma} \dot{\varepsilon} d \sigma
$$

being responsible for the energy dissipation during hot working through a microstructural change of the material. Therefore, the dissipation function in relation to the plastic flow stability is represented by $J$ in the framework of DMM and the dissipation function of Ziegler, $D$ is substituted by $J$, being $D=J$. After some pertinent substitutions, as detailed in Reference 9, the so-called Ziegler-Prasad criterion is obtained:

$$
\frac{\partial J}{\partial \dot{\varepsilon}}>\frac{J}{\dot{\varepsilon}}
$$

This equation can be formulated in the form:

$$
\frac{\partial \operatorname{Ln}(J)}{\partial \operatorname{Ln}(\dot{\varepsilon})}>1
$$

or also in a form that is interesting for this work:

$$
\frac{\partial J}{\partial \dot{\varepsilon}}-\frac{J}{\dot{\varepsilon}}>0
$$

This is therefore the criterion developed by Prasad and Kumar to study stability that is also called the Ziegler-Prasad criterion (Eqs. [6] and [8] are different forms of the same criterion). Up to here, the criterion can be considered as valid. The problem arises when concrete constitutive equations for deformation are used to have a practical criterion for stability. Our model, which will be described later, arises from Eq. [8], 
defining a coefficient rho that is derived from the application of DMM to the Ziegler theory by Kumar, Prasad et al. in the form:

$$
\rho=\frac{\partial J}{\partial \dot{\varepsilon}}-\frac{J}{\dot{\varepsilon}}
$$

with the condition for the stability $\rho>0$. This equation is valid for a power law, for Garofalo or for other constitutive equation, but none is superior to the Garofalo equation in their capability to fit large ranges of experimental data of $T, \sigma$ and $\dot{\varepsilon} .^{[14,32-36]}$

The condition of Eq. [9] is defined before any constitutive equation that could be used to describe the thermomechanical process. To obtain a practical expression for $J$ and $G$, Kumar and Prasad, and others, used a power law since it is necessary to define a concrete constitutive equation.

The attainment of the stability expression of Prasad, or the Xi equation, from the concepts of the DMM and the use of a power law equation is given in Appendix A. This expression,

$$
\xi(\dot{\varepsilon})=\frac{\partial\left(\ln \left(\frac{m}{m+1}\right)\right)}{\partial \ln (\dot{\varepsilon})}+m<0,
$$

is given in most of the hot-working publications that deal with stability studies ${ }^{[37-42]}$ and will be substituted in our model by the stability parameter rho as will be shown later.

It is considered that for $\xi(\dot{\varepsilon})<0$ microstructural instabilities are present during hot working. However, strictly, $m=m^{P L}$ has a constant value, and since $\frac{\partial\left(\ln \left(\frac{m}{m+1}\right)\right)}{\partial \ln (\dot{\varepsilon})}=0$ then $m<0$. This is an inconsistency because experimentally $m$ is always higher than zero. Other inconsistencies were pointed out by other authors such as Montheillet et al. ${ }^{[43]}$ Murty et al. ${ }^{[11]}$ and Ghosh. ${ }^{[4]}$

To avoid the inconsistency of the constant value of $m$ with thermomechanical variables, Prasad et al., ${ }^{[21,28-30]}$ among others, divide or chop the variable range in small terms which implies large potential numeric errors. They impose a variability of $\mathrm{m}$, creating polynomial constitutive equations without a physical basis.

\section{PROPOSED MODEL OF STABILITY THROUGH THE USE OF A GAROFALO EQUATION}

As mentioned, the use of a power law has important inconsistencies. One way to avoid this is by using a Garofalo equation that will allow a dependency of $m$ with $T$ and $\dot{\varepsilon}$ in a natural way. This is the equation that we have chosen as the best of the equations susceptible to being a constitutive equation, with physical sense and without the use of "ad hoc" polynomials. On the other hand, this necessarily implies rejecting the widespread use of power laws.

The supremacy of the Garofalo equation is due to the ability to obtain information of the material processing by means of its parameters. ${ }^{[4]}$ This is because, despite being a phenomenological equation, it has a strong theoretical basis as discussed by Rieiro et al. ${ }^{[14,33,34]} \mathrm{It}$ also has a high capacity to represent the phenomena of plastic deformation by hot working for wide ranges of stress, temperature and strain rates. ${ }^{[14,32-36]}$ This equation is also proposed by many authors when analyzing a wide range of stress values. ${ }^{[22,23,39,40,46]}$

The Garofalo equation has the form:

$$
\dot{\varepsilon}=A^{G} \cdot e^{-\frac{Q^{G}}{R \cdot T}} \cdot\left[\sinh \left(\alpha^{G} \cdot \sigma\right)\right]^{n^{G}}
$$

where $\left\{A^{G}, Q^{G}, \alpha^{G}, n^{G}\right\}$ are constant parameters at a given value of strain $\varepsilon$, and the only thermomechanical variables involved in the equation are $\{\dot{\varepsilon}, T, \sigma\}$. This equation is adequate for a given value of strain, or for steady state (when it is present). Usually, the maximum or peak stress value is used, as shown in various works, but it is also possible to take data values for different strains. ${ }^{[5,14,32-35]}$

The original Garofalo equation is not dependent on $\varepsilon$ but the validity of the equation at various strains has been proved, ${ }^{[5,32-35]}$ extending the equation to be a deformation-dependent constitutive equation of the form:

$$
\dot{\varepsilon}=A^{G}(\varepsilon) \cdot e^{-\frac{Q^{G}(\varepsilon)}{R \cdot T}} \cdot\left[\sinh \left(\alpha^{G}(\varepsilon) \cdot \sigma\right)\right]^{n^{G}(\varepsilon)}
$$

where $\{\sigma, T, \dot{\varepsilon}, \varepsilon\}$ are the thermodynamic variables involved in processing and $\left\{A^{G}(\varepsilon), Q^{G}(\varepsilon), \alpha^{G}(\varepsilon), n^{G}(\varepsilon)\right\}$ are the Garofalo parameters that depend on strain. The index $G$ stands for Garofalo in order to differentiate the equations involving a power law (PL).

The experimental data at a given strain can be easily adjusted using the Garofalo equation as the best adjusted function that minimizes the adjustment errors by the methods of non-linear least squares. This will allow us to obtain forming maps for each strain. The method is simple and efficient, with adequate statistical support to assess the quality of the results. The priority and primacy of the Garofalo equation are emphasized by many authors, ${ }^{[22,23,46]}$ among others.

The use of the Garofalo equation, as will be seen later, leads to the construction of contour maps for functions that evaluate stability or efficiency, in a continuous form. The maps do not have discontinuity or interpolation error inter-zones or calculation errors.

To develop an algorithm for the rho parameter, defined in Eq. [9], it was previously necessary to define the best expression for the dissipator co-content, $J$, and the best procedure for the calculation of the derivative, $d J / d \dot{\varepsilon}$, at constant strain and temperature. The calculation of $J$ and the efficiency, $\eta$, using the Garofalo equation, requires operating with complicated integral expressions or derivative expressions. The use of an algorithm is a common praxis in this kind of mathematical problems in order to simplify these operations. This will also help to reach an expression for the stability control parameter rho, $\rho$, in our new model, which can be compared to Eq. [10]. In this work, an algorithm optimum for the given experimental stresses, strain rates and temperatures is used. Three more algorithms have been tried to verify the quality of the 
numerical or analytical results, and all verifications have been satisfactory.

From $J=\int_{0}^{\sigma} \dot{\varepsilon} d \sigma$, the following variable change can be made: $x=\alpha^{G} \cdot \sigma$. It is then obtained:

$$
J=\int_{0}^{\alpha^{G} \cdot \sigma} \dot{\varepsilon} \frac{\mathrm{d} x}{\alpha^{G}}=\int_{0}^{\alpha^{G} \cdot \sigma} A^{G} \cdot e^{-\frac{Q^{G}}{R \cdot T}} \cdot\left[\sinh \left(\alpha^{G} \cdot \sigma\right)\right]^{n^{G}} \cdot \frac{\mathrm{d} x}{\alpha^{G}}
$$
form:

This expression can be rewritten in the following

$$
J=\left(\frac{A^{G} \cdot e^{-\frac{Q^{G}}{R \cdot T}}}{\alpha^{G}}\right) \int_{0}^{\sinh ^{-1}\left(\theta(\dot{\varepsilon}, T)^{G}\right)}[\sinh (x)]^{n^{G}} \cdot \mathrm{d} x
$$

where $\left(\theta(\dot{\varepsilon}, T)^{G}\right)=\left(\frac{\dot{\varepsilon} \cdot e^{\frac{Q^{G}}{R \cdot T}}}{A^{G}}\right)^{\left(1 / n^{G}\right)}$. This expression is introduced by its utility for the calculation of results in the plane $\{\dot{\varepsilon}, T\}$ and is similar to the expression $\left(\theta^{G}\right)=$ $\left(\frac{Z}{A}\right)^{\frac{1}{n}}$ used by Wang et al. ${ }^{[22,23]}$ For $Z=Z(\dot{\varepsilon}, T)$, then $\left(\theta(\dot{\varepsilon}, T)^{G}\right)=\left(\frac{Z(\dot{\varepsilon}, T)}{A}\right)^{\frac{1}{n}}$.

Notably, the identity $\alpha^{G} \cdot \sigma=\sinh ^{-1}\left(\theta(\dot{\varepsilon}, T)^{G}\right)$ has been used. On the other hand, it is evident that, with slight equivalent algebraic transformations (external to the integral), the calculation of $J / \dot{\varepsilon}$, the second term of Eq. [9], leads to $\left(\theta(\dot{\varepsilon}, T)^{G}\right)^{n^{G}}=\left(\frac{\dot{\varepsilon} \cdot e^{\frac{Q^{G}}{R \cdot T}}}{A^{G}}\right)$ and therefore:

$$
\begin{aligned}
J / \dot{\varepsilon} & =\left(\frac{A^{G} \cdot e^{-\frac{Q^{G}}{R \cdot T}}}{\alpha^{G} \cdot \dot{\varepsilon}}\right) \int_{0}^{\sinh ^{-1}\left(\theta(\dot{\varepsilon}, T)^{G}\right)}[\sinh (x)]^{n^{G}} \cdot \mathrm{d} x \\
& =\left(\frac{1}{\alpha^{G} \cdot\left(\theta(\dot{\varepsilon}, T)^{G}\right)^{n^{G}}}\right) \cdot \int_{0}^{\sinh ^{-1}\left(\theta(\dot{\varepsilon}, T)^{G}\right)}[\sinh (x)]^{n^{G}} \cdot \mathrm{d} x
\end{aligned}
$$

giving:

$$
J / \dot{\varepsilon}=\left(\frac{1}{\alpha^{G} \cdot\left(\theta(\dot{\varepsilon}, T)^{G}\right)^{n^{G}}}\right) \cdot \int_{0}^{\sinh ^{-1}\left(\theta(\dot{\varepsilon}, T)^{G}\right)}[\sinh (x)]^{n^{G}} \cdot \mathrm{d} x
$$

Once $J$ is determined, the calculation of the efficiency $\eta$ is straightforward since:

$$
\eta=\frac{J}{J_{\max }}=\frac{J}{\left(\frac{\dot{\varepsilon} \cdot \sigma}{2}\right)}=\frac{2 \cdot J}{\dot{\varepsilon} \cdot \sigma}
$$

Every expression of $J$ will lead to an expression associated with the efficiency.

$$
\begin{aligned}
\eta^{G} & =\frac{2 \cdot\left(\frac{A^{G} \cdot e^{-\frac{Q^{G}}{\alpha^{G}}}}{\alpha^{G}}\right) \int_{0}^{\sinh ^{-1}\left(\theta(\dot{\varepsilon}, T)^{G}\right)}[\sinh (x)]^{n^{G}} \cdot \mathrm{d} x}{\dot{\varepsilon} \cdot \frac{1}{\alpha^{G}} \cdot \sinh ^{-1}\left[\theta(\dot{\varepsilon}, T)^{G}\right]} \\
= & \frac{2 \cdot \int_{0}^{\sinh ^{-1}\left(\theta(\dot{\varepsilon}, T)^{G}\right)}[\sinh (x)]^{n^{G}} \cdot \mathrm{d} x}{\left(\theta(\dot{\varepsilon}, T)^{G}\right)^{n^{G}} \cdot \sinh ^{-1}\left[\theta(\dot{\varepsilon}, T)^{G}\right]}
\end{aligned}
$$

Once the parameters $J$ and $\eta$ are calculated, it is possible to obtain the stability parameter rho that substitutes the parameter xi, in the equation of Prasad, Eq. [10].

As observed in Eq. [9], $\rho=\frac{\partial J}{\partial \dot{\varepsilon}}-\frac{J}{\dot{\varepsilon}}$. A derivation of the term $\frac{\partial J}{\partial \dot{\varepsilon}}$ (for a given strain and temperature) is given in Appendix B as:

$$
\frac{\partial J}{\partial \dot{\varepsilon}}=\frac{1}{n^{G} \cdot \alpha^{G}} \cdot \frac{\theta(\dot{\varepsilon}, T)^{G}}{\sqrt{1+\theta(\dot{\varepsilon}, T)^{G^{2}}}}
$$

On the other hand, the term $\frac{J}{\dot{\varepsilon}}$ is given in Eq. [15]. It is finally reached:

$$
\begin{aligned}
\rho= & \frac{1}{n^{G} \cdot \alpha^{G}} \cdot\left[\frac{\theta(\dot{\varepsilon}, T)^{G}}{\sqrt{1+\theta(\dot{\varepsilon}, T)^{G^{2}}}}-\left(\frac{n^{G}}{\left(\theta(\dot{\varepsilon}, T)^{G}\right)^{n^{G}}}\right)\right. \\
& \left.\cdot \int_{0}^{\sinh ^{-1}\left(\theta(\dot{\varepsilon}, T)^{G}\right)}[\sinh (x)]^{n^{G}} \cdot \mathrm{d} x\right]>0
\end{aligned}
$$

The developed calculation algorithm presents two parts. A first one, between the square brackets, has been fully analytically developed. The other involves the calculation of an integral for each value of $\{\dot{\varepsilon}, T\}\}$. Since $n^{G}$ is a real number, the analytic calculation of the integral containing this number is a difficult task. Momeni et al. ${ }^{[47]}$ use a hypergeometric function to solve the problem but they do not find an explicit analytic expression. Therefore, it is convenient to use numerical calculations through current programs as MATLAB, Mathcad and others. The steps to reach this expression that will be used to draw stability maps are given in Appendix C.

\section{METHODOLOGICAL ASPECTS AND IMPLEMENTATION OF SOFTWARE}

To define the behavior of materials in the hot-forming process, it is necessary to obtain safe and reliable parameters of the Garofalo constitutive equation. In numerous works, like that of Hamada et al., ${ }^{[4]}$ the complicated process that may be used to obtain the parameters of the Garofalo equation by means of adjustments by steps in the framework of linear processes can be observed; this is typical of a power law approximation. Also, Babu et al. ${ }^{[4]}$ use the Garofalo 
constitutive equation but the parameters are obtained by a power law approximation. This leads to wrong values of the activation energy or the pre-exponential factor as shown by Wang et al. ${ }^{[50]}$

In this work, the parameters of the Garofalo equation, $\{A, Q, n, \alpha\}$, are determined by a non-linear method involving an algorithm specifically developed for the treatment of this equation, named the RCR (Rieiro-Carsi-Ruano) method described previously. ${ }^{[5,14,32-34]}$ This method allows for an automatic procedure divided into several steps in MATLAB programs for calculation of the values of this equation for any material.

A new criterion for the efficiency and stability for hot working of metallic materials based on the Ziegler-Prasad criterion is introduced in this investigation. This stability criterion is given by the equation $\frac{d J}{d \dot{\varepsilon}}-\frac{J}{\dot{\varepsilon}}>0$.

In the work relative to the Lyapunov criterion, ${ }^{[5]}$ we compared our results on the magnesium alloy AZ31 with those of other authors on the same alloy. In the present work, we preferred to use data of other authors and have a direct comparison of their stability, xi, criterion with our new, rho, criterion introducing the Garofalo equation. Notably, both criteria are based on the criterion deduced within the framework of the Ziegler theory and in the framework of the DMM.

Other particularly important methodological aspects are those that involve the implementation of calculation algorithms in efficient programs and the methodology used to validate and evaluate the results. We used MATHCAD v. 7.0 software to implement the algorithms for calculation of the efficiency as well as to build stability maps. This software is simple and effective, and graphic representations are easy to obtain.

\section{RESULTS}

Compression Tests from the Work of Balasubrahmanyam and Prasad ${ }^{[24]}(\mathrm{B}-\mathrm{P})$ on Ti-10V-4.5Fe-1.5Al alloy were chosen to compare the stability maps by the two mentioned models: that of Prasad et al. ${ }^{[20,21]}$ and our model incorporating a Garofalo equation. In the work of B-P, data were obtained in the form of tables at a large range of strain rates, from 0.001 to $100 \mathrm{~s}^{-1}$, and temperatures, $650{ }^{\circ} \mathrm{C}$ to $900{ }^{\circ} \mathrm{C}(923 \mathrm{~K}$ to $1173 \mathrm{~K})$, which allow a fair comparison. Cylindrical specimens of $10 \mathrm{~mm}$ diameter and $15 \mathrm{~mm}$ height were machined from the hot-rolled rod. The specimens were solution annealed at $765{ }^{\circ} \mathrm{C}$ for $30 \mathrm{~min}$ and water quenched. The microstructure consisted of single-phase grains of about $60 \mu \mathrm{m}$ in size. The $\mathrm{B}-\mathrm{P}$ data are tabulated at strains of 0.1 to 0.5 . At each strain 36 data points are given. This is an adequate amount of data to perform a statistic of control errors. The data at strains of 0.2 and 0.4 are given in Table I.

These strains were chosen by Balasubrahmanyam and Prasad $^{[24]}$ to draw their maps, and for comparison we calculate our maps for the same strains. At 0.2 the material has just passed the peak stress and at 0.4 enters into steady state and shows a better statistical quality fit than at other strains.
The RCR method (in the MATLAB code and/or QBASIC code $)^{[14,32-36]}$ was applied to these data to obtain the parameters of the Garofalo equation. This method also gives the parameters controlling the quality and precision of the adjustment. The results are given in Table II.

The statistical parameters of the table refer to the coefficient of determination, $R^{2}$, the Fisher-Snedecor parameter, $F$, the sum of square errors, SSE, and the variance, $\sigma^{2}$. These parameters are very significant, and therefore the adjustment can be considered as very good.

The stability map using the Garofalo equation and the parameter rho (Eq. [19]) is given in Figure 2 at $\varepsilon=$ 0.2 and 0.4 .

In Figure 2, the evolution of $\rho$ is plotted against the temperature and the strain rate. When the units of $\sigma$ are $\mathrm{MPa}$ and those of $\dot{\varepsilon}$ are $\mathrm{s}^{-1}$, the units of rho are $[\rho]=\frac{\text { Energy }}{\text { Volume }}$; in our case, the units are Joule $/ \mathrm{m}^{3}$.

The figure shows the more stable region, which is that situated between the two lines of the rho value equal to 7.5 at $\varepsilon=0.2$ and 5.5 at $\varepsilon=0.4$. The optimum thermomechanical conditions for hot working this material given as a dotted line is situated in the center of the more stable band. The band of greater safety or greater potential for stability has an amplitude of $80^{\circ} \mathrm{C}$ to $130{ }^{\circ} \mathrm{C}$ in temperature $(80 \mathrm{~K}$ to $130 \mathrm{~K})$. This band is established by a functional relationship between the logarithm of the strain rate (in $\mathrm{s}^{-1}$ ) and the temperature (in ${ }^{\circ} \mathrm{C}$ or $\mathrm{K}$ ) in the following form for $\varepsilon=0.2$ :

$$
\begin{aligned}
\log _{10}(\dot{\varepsilon})= & -0.000008436 \cdot T^{2}+0.024924924 \cdot T \\
& -14.237789312
\end{aligned}
$$

The relation is parabolic and significantly marked with $R^{2}=0.9998$. This gives an optimum stability temperature of $775^{\circ} \mathrm{C}(1048 \mathrm{~K})$ for $1 \mathrm{~s}^{-1}$.

For the strain of $\varepsilon=0.4$ :

$$
\begin{aligned}
\log _{10}(\dot{\varepsilon})= & -0.000010327 \cdot T^{2}+0.026502485 \cdot T \\
& -14.974929664
\end{aligned}
$$

In this case, $R^{2}=0.9997$. This gives for $1 \mathrm{~s}^{-1}$ an optimum stability temperature of $840{ }^{\circ} \mathrm{C}(1113 \mathrm{~K})$.

There are no markedly unsafe areas or areas susceptible to instability in front of a punctual disturbance, but the two triangular regions situated in the two corners (upper left and lower right), colored in red, are the less stable regions in the map. The area in the upper left corner, at high strain rates and low temperatures, is susceptible to developing processes of adiabatic shear bands or of ductile fracture due to formation of voids according to Figure 1. In addition, the small area in the lower right corner is a zone of less stability, which is susceptible to reaching instability in the event of disturbances, punctual or local, due to wedge cracking.

Regarding the efficiency, the maps obtained at $\varepsilon=0.2$ and 0.4 by Eq. [17] are given in Figure 3. The maps show that, for a given strain rate, the efficiency increases with an increase of temperature, which is typical of all 
Table I. Data of Balasubrahmanyam and Prasad ${ }^{[24]}$ for the Ti-10V-4.5Fe-1.5Al Alloy at $\varepsilon=0.2$ and 0.4

\begin{tabular}{|c|c|c|c|c|c|c|c|}
\hline$T\left({ }^{\circ} \mathrm{C}\right)$ & $\varepsilon\left(\mathrm{s}^{-1}\right)$ & $\begin{array}{c}\sigma(\mathrm{MPa}) \\
\varepsilon=0.2\end{array}$ & $\begin{array}{c}\sigma(\mathrm{MPa}) \\
\varepsilon=0.4\end{array}$ & $T\left({ }^{\circ} \mathrm{C}\right)$ & $\hat{\varepsilon}\left(\mathrm{s}^{-1}\right)$ & $\begin{array}{c}\sigma(\mathrm{MPa}) \\
\varepsilon=0.2\end{array}$ & $\begin{array}{c}\sigma(\mathrm{MPa}) \\
\varepsilon=0.4\end{array}$ \\
\hline 650 & 0.001 & 110.6 & 102.7 & 800 & 0.001 & 27.9 & 28.3 \\
\hline 650 & 0.01 & 212.3 & 176.2 & 800 & 0.01 & 59.3 & 59.0 \\
\hline 650 & 0.1 & 332.2 & 269.7 & 800 & 0.1 & 118.9 & 117.7 \\
\hline 650 & 1.0 & 498.3 & 370.8 & 800 & 1.0 & 206.4 & 202.9 \\
\hline 650 & 10.0 & 681.7 & 548.8 & 800 & 10.0 & 319.0 & 308.7 \\
\hline 650 & 100.0 & 882.7 & 669.4 & 800 & 100.0 & 420.1 & 396.5 \\
\hline 700 & 0.001 & 70.8 & 64.6 & 850 & 0.001 & 21.8 & 22.1 \\
\hline 700 & 0.01 & 132.7 & 125.9 & 850 & 0.01 & 43.0 & 44.0 \\
\hline 700 & 0.1 & 224.9 & 213.2 & 850 & 0.1 & 85.6 & 85.0 \\
\hline 700 & 1.0 & 339.1 & 284.1 & 850 & 1.0 & 168.5 & 163.5 \\
\hline 700 & 10.0 & 541.2 & 447.5 & 850 & 10.0 & 267.4 & 254.1 \\
\hline 700 & 100.0 & 679.1 & 550.9 & 900 & 100.0 & 350.7 & 341.6 \\
\hline 750 & 0.001 & 47.1 & 45.7 & 900 & 0.001 & 17.8 & 18.4 \\
\hline 750 & 0.01 & 80.6 & 79.2 & 900 & 0.01 & 35.0 & 35.9 \\
\hline 750 & 0.1 & 155.1 & 152.3 & 900 & 0.1 & 69.3 & 69.0 \\
\hline 750 & 1.0 & 252.8 & 233.1 & 900 & 1.0 & 140.6 & 136.1 \\
\hline 750 & 10.0 & 439.5 & 372.2 & 900 & 10.0 & 221.5 & 213.1 \\
\hline 750 & 100.0 & 536.1 & 462.0 & 900 & 100.0 & 279.9 & 281.8 \\
\hline
\end{tabular}

Table II. Garofalo Parameters and Statistics of the Adjustment of the Data of Balasubrahmanyam and Prasad ${ }^{[2]}$ at $\varepsilon=0.2$ and 0.4 for the Ti-10V-4.5 Fe-1.5Al Material

\begin{tabular}{lll}
\hline Parameters & \multicolumn{1}{c}{$\begin{array}{c}\text { Values } \\
\varepsilon=0.2\end{array}$} & \multicolumn{1}{c}{$\begin{array}{c}\text { Values } \\
\varepsilon=0.4\end{array}$} \\
\hline$A\left(\mathrm{~s}^{-1}\right)$ & $4.440237620 \mathrm{E}+12$ & $4.2010252 \mathrm{E}+10$ \\
$\ln (A)$ & 29.12172901 & 24.4611795 \\
$Q(\mathrm{~kJ} / \mathrm{mol})$ & 249.061 & 222.489 \\
$n$ & 3.710 & 3.556 \\
$\alpha\left(\mathrm{MPa}^{-1}\right)$ & 0.00338 & 0.005060 \\
$R^{2}$ & 0.989 & 0.99365 \\
$F($ exper $)$ & 952.169 & 1669.665 \\
$F($ theor $)$ & 2.901 & 2.901 \\
$\mathrm{SSE}$ & 0.193 & 0.11043 \\
$\sigma^{2}$ & 0.193 & 0.11043 \\
\hline
\end{tabular}

physical systems. On the other hand, for a given temperature, the efficiency increases for decreasing strain rates, which is in agreement with the fluid and plastic flow processes.

For comparison, an adapted version of the processing maps of $\mathrm{B}-\mathrm{P}^{[24]}$ (Figure 6(a) through (b) in their work), obtained by application of Eq. [10], is given in Figure 4 at $\varepsilon=0.2$ and 0.4 . The maps are based on the same Ziegler-Prasad criterion as that used in our rho model. The difference is that the model applied by $\mathrm{B}-\mathrm{P}$ used a power law to arrive to the xi stability condition, and we used the Garofalo equation to arrive at the rho stability condition.

Notably, the contours of Figure 4 represent constant efficiency in percent while those of Figure 3 represent constant efficiency marked per unit. B-P superimpose a region that they define as unstable on the efficiency map of Figure 4, without qualifying it, which is between about $670{ }^{\circ} \mathrm{C}$ to $900{ }^{\circ} \mathrm{C}$ and 7 to $100 \mathrm{~s}^{-1}$. The authors show overlapping areas of instability but they do not qualify the rest of the areas in the map. We consider that these large areas have zones that are more or less safe or, according to their description, more or less unstable. However, the authors do not perform a quantification. In other words, the work does not provide the necessary information to contrast the contour lines of the parameter xi, (Eq. [10]), which measures the stability, with the contour lines of the parameter that measures the efficiency.

This approach to obtaining the forming maps as an overlap of the efficiency and the stability, but only pointing out some significant areas or domains, is common in the literature. ${ }^{[51]}$ A complete review including the power law methodology and the use of constitutive equations of the polynomial type of order three adjusted to the stress is given in the work of Zhang et $a$. $^{\text {[52] }}$

In contrast, our stability maps (Figure 2) identify the safest areas as those that reach the stability condition with most intensity. In addition, the rho parameter is modeled with its corresponding units, being able to relate these values to the inequality that defines the stability. We qualify as less safe or less stable zones those that are close to zero. This is an absolute novelty in the capacity of predicting the forming behavior of materials.

On the other hand, the unstable region between 670 ${ }^{\circ} \mathrm{C}$ and $900{ }^{\circ} \mathrm{C}$ (943 to $1173 \mathrm{~K}$ ) for strain rates of about 10 to $100 \mathrm{~s}^{-1}(\log 1$ to $\log 2)$ determined by $\mathrm{B}-\mathrm{P}$ (Figure 4) does not agree with the less stable of our maps in Figure 2. Furthermore, most of its unstable area is in an area on our maps that is identified as reasonably stable, with high values of the rho parameter. This is in agreement with the studies of Raj ${ }^{[16,17]}$ and Raghunhat et $a l .{ }^{[18]}$ (Figure 1) that predict dynamic recrystallization processes that develop in a stable manner. 

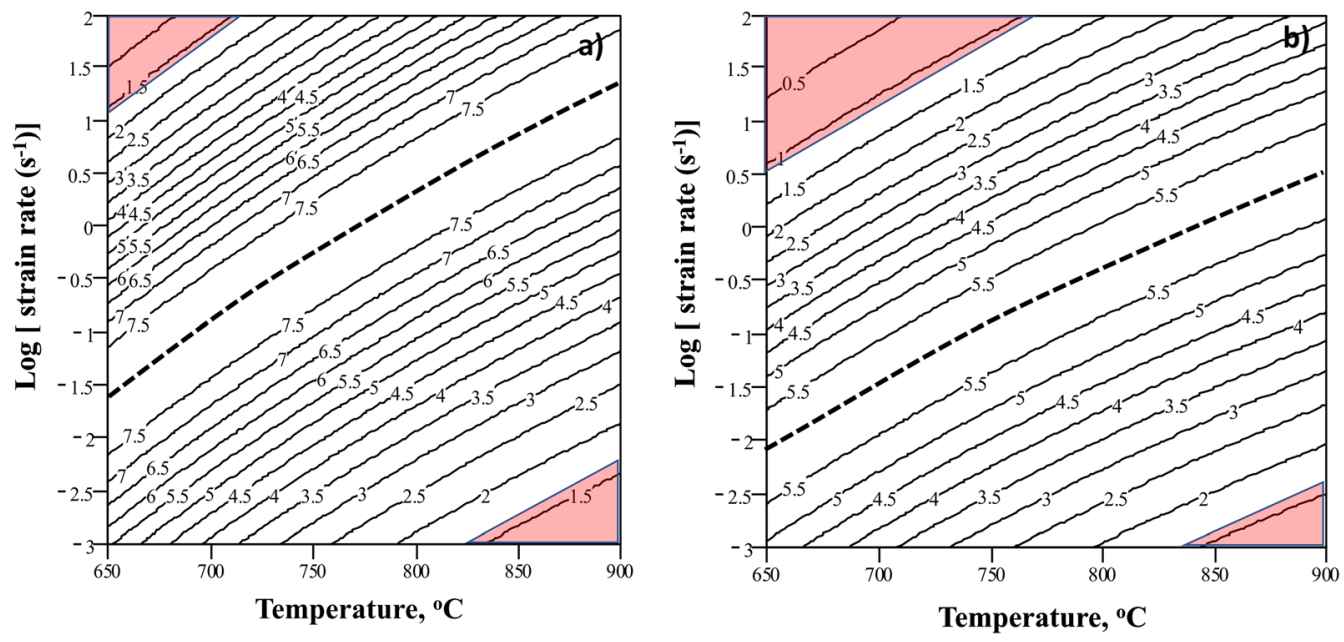

Fig. 2-Stability map using the Garofalo equation and the rho parameter for the R2 criterion for the alloy Ti-10V-4.5Fe-1.5Al obtained using data from Table I, ${ }^{[24]}(a)$ at $\varepsilon=0.2$ and $(b)$ at $\varepsilon=0.4$. The dotted line corresponds to the more stable conditions.

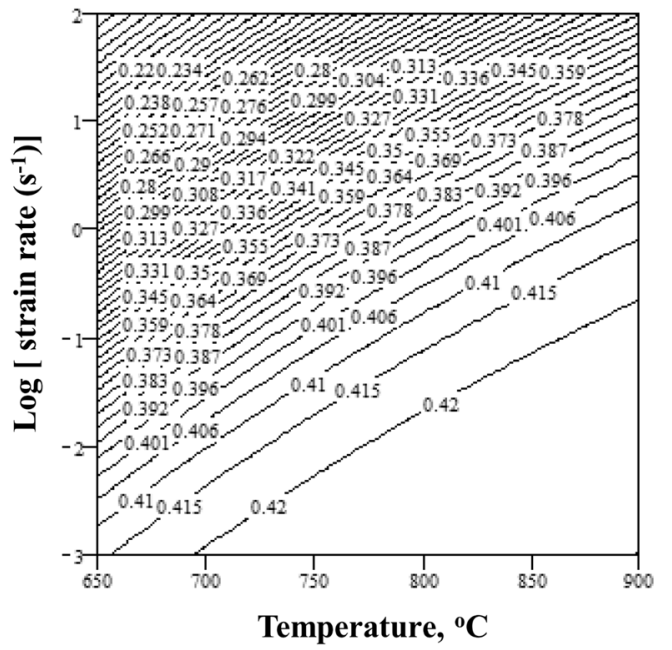

(a)

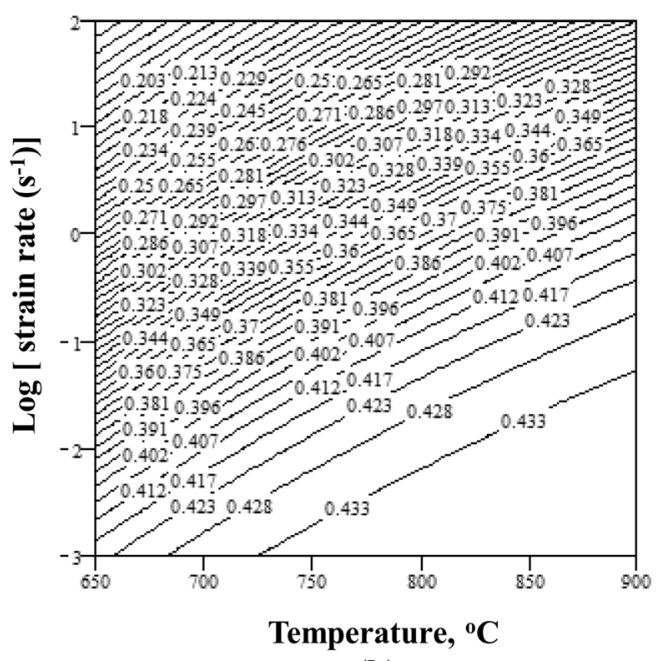

(b)

Fig. 3-Efficiency map for the alloy Ti-10V-4.5Fe-1.5Al obtained using data from Table I. [24] at $(a) \varepsilon=0.2$ and $(b) \varepsilon=0.4$. The number over the contour lines gives the efficiency value expressed in parts per unit.

In addition, their maps in Figure 4 show a peak of efficiency at about $860^{\circ} \mathrm{C}(1133 \mathrm{~K})$ and $10^{-2} \mathrm{~s}^{-1}$. In our map in Figure 2, that peak is not present, which, on the other hand, is reasonably expected. That peak in their work implies that higher efficiencies can be achieved by reducing temperatures, which in this context does not seem reasonable.

In absolute values, the difference between their efficiencies at $\varepsilon=0.2$ and those of our work, at maximum, differs from 48 to 42 pct. This difference of about 6 pct, which is about the same at $\varepsilon=0.4$, is understandable owing to the interpolation errors. This is due to the partial adjustment or fit of the strain rate sensitivity, $\mathrm{m}$, using power laws by zones or intervals and then pretending to build a single map with the values of the different zones. Those interpolation errors can easily reach values of 5 to 7 pct. The first problem is that a partial adjustment of data by third order polynomials, or with different values for $Q, n$ and $A$ (parameters that defined the power law equation), is very difficult. These polynomials have to be forced to converge at a point on the border of two regions. This disagreement is one of the main sources of errors. The modeling with the power law equation, together with the use of third-degree interpolation polynomials, could explain the differences between their and our maps.

It is interesting to compare our efficiency maps in Figure 3 obtained at $\varepsilon=0.2$ (a) and at $\varepsilon=0.4$ (b). The maximum and minimum values given in the maps at $\varepsilon=$ 0.2 are 0.42 and 0.22 , respectively, in contrast to 0.43 and 0.20 at $\varepsilon=0.4$. In Figure 4(a) at $\varepsilon=0.2$, the maximum and minimum values in efficiency are 0.48 and 0.12 , and in Figure 4(b) at $\varepsilon=0.4$, the maximum and minimum values in efficiency are 0.46 and 0.14 . It is then concluded that the differences are small between deformation 0.2 and deformation 0.4 , both in the work of 


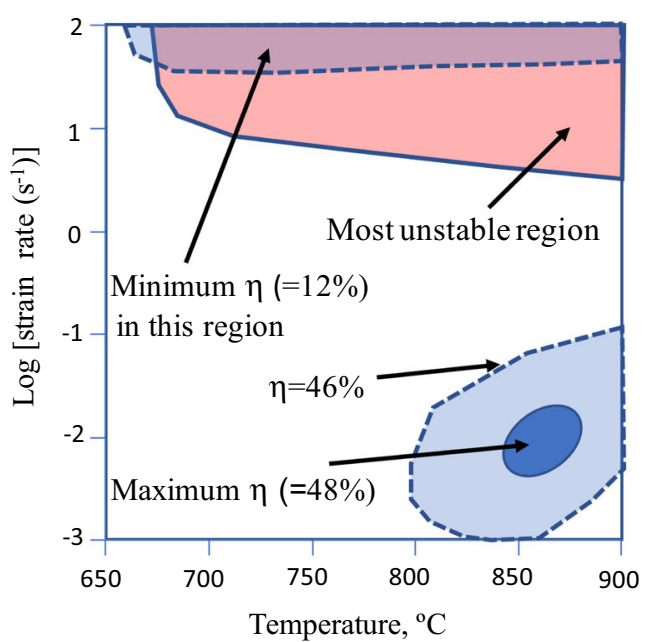

(a)

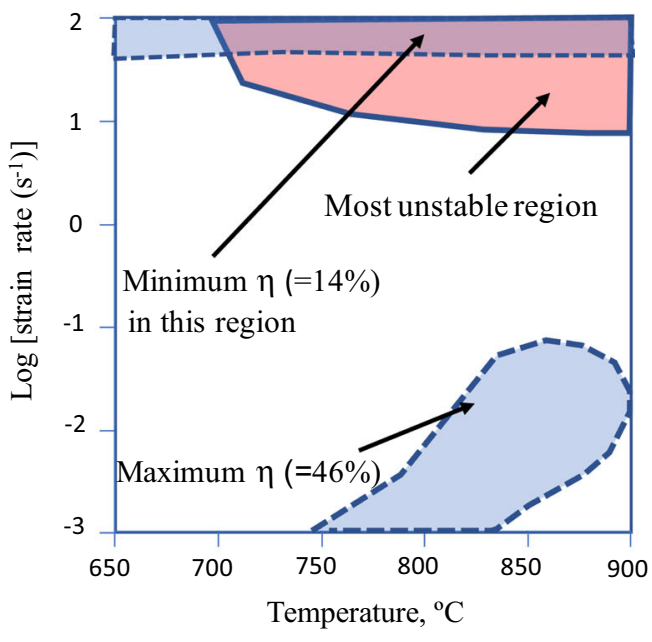

(b)

Fig. 4-Adapted version of the processing maps of Balasubrahmanyam and Prasad ${ }^{[24]}$ (Fig. 6(a) through (b) in their work) for the Ti-10V-4.5Fe-1.5Al alloy at $(a) \varepsilon=0.2$ and $(b) \varepsilon=0.4$. The numbers on the isolines correspond to the efficiency in percent. The regions limited by thick continuous lines are regions of instability, and the regions limited by thick dashed lines are regions of maximum or minimum efficiency, $\eta$.

B-P and in ours. The differences in efficiencies between the two works are small if the island of efficiency, 0.48 , in Figure 4(a), which has a difficult physical justification, is not considered. The percentage of differences in maxima between the work of $\mathrm{B}-\mathrm{P}$ and ours is 5 and 10 pct for deformations of 0.4 and 0.2 , respectively. In the minimum, the percentage of differences is 11 and 17 pct for deformations of 0.4 and 0.2 , respectively.

Regarding the instability, the maps of $\mathrm{B}-\mathrm{P}^{[24]}$ (Figure 4), which were built using a power law for the calculation of $\eta$ and $J$, provide little information. Almost the same instability zones are present at $\varepsilon=$ 0.2 and 0.4 . In both cases the maps show quasi-rectangular areas. For $\varepsilon=0.2$ the rectangle has sides ( 0.8 to 2 ) in $\log 10$ of the strain rate and from $670{ }^{\circ} \mathrm{C}$ to $900{ }^{\circ} \mathrm{C}$ $(943 \mathrm{~K}$ to $1173 \mathrm{~K})$, and for deformation (0.4) the rectangle has sides ( 0.8 to 2$)$ in $\log 10$ of the strain rate and $700{ }^{\circ} \mathrm{C}$ to $900{ }^{\circ} \mathrm{C}(973 \mathrm{~K}$ to $1173 \mathrm{~K})$.

In contrast, our maps in Figure 2 that were built from Eqs. [17] and [19] provide a large amount of information regarding the instability at these two strains that is obtained from the evaluation of the rho parameter in the $R^{2}$ model. Figure 2 shows that the maximum value of the rho parameter at $\varepsilon=0.2$ is larger than at $\varepsilon=0.4$ (7.97 vs. 5.78). This means that the zone of higher stability (around the dotted line) is safer at $\varepsilon=0.2$ than at $\varepsilon=0.4$ since the rho parameter has decreased by 27 pct. The figure also shows that the minimum value of the rho parameter at $\varepsilon=0.2$ is larger than at $\varepsilon=0.4(0.58 \mathrm{vs}$. 0.20$)$, which indicates that at this strain the less stable zone may become unstable more easily. On the other hand, a comparison of our stability maps at $\varepsilon=0.2$ and 0.4 in this figure allows us to conclude that the maximum stability temperature, at a given strain rate, at $\varepsilon=0.4$ is higher than at $\varepsilon=0.2$. For instance, at $\dot{\varepsilon}=1 \mathrm{~s}^{-1}$ the maximum stability temperature is $775^{\circ} \mathrm{C}(1048 \mathrm{~K})$ at $\varepsilon$ $=0.2$ while at $\varepsilon=0.4$ it is $835^{\circ} \mathrm{C}(1108 \mathrm{~K})$.
Notably, there is no coincidence of the maps in Figure 4 with our maps regarding the zone of instability, or with the sense of the evolution of this instability. It is difficult to assume that their zone of stability is homogeneously uniform differentiating only the best working area in their efficiency map.

It has to be also noted that we have adjusted the Garofalo equation with the parameters given in Table II for $\varepsilon=0.2$ and 0.4 . At each of these strains, the adjustment has high statistical quality, and the values may be applied at all ranges of the thermomechanical variables. In contrast, the work of $\mathrm{B}-\mathrm{P}^{[24]}$ uses a power law with the parameters $A, n$ and $Q$ that are dependent on the temperature and strain rate but not dependent on the deformation, as may be expected from a constitutive equation. The authors find values of $n$ of about 3 below $0.1 \mathrm{~s}^{-1}$ and about 8 at high strain rates. For this reason, the activation energy is strongly influenced by the stress chosen being $183 \mathrm{~kJ} / \mathrm{mol}$ below $250 \mathrm{MPa}$ and $398 \mathrm{~kJ} / \mathrm{mol}$ at high stresses. Once again, the arbitrary partitions of these authors can cause large numeric errors because of the use of polynomials for adjusting each interval of application of the power law and applying interpolation algorithms that have to join values of $n$ or $m$ of different zones. Therefore, the contour lines of the maps must be forced, providing situations of difficult assimilation in the continuity of the material processes.

Despite the differences between the two methods, there are also important coincidences. Their stability zones, indicated as most stable, have a 70 to 80 pct coincidence with our most secure zones, although they do not qualify the differential quality of the stability.

\section{CONCLUSIONS}

(1) A new model of stability is proposed through the introduction of the Garofalo equation in the 
general criterion of Ziegler-Prasad all under the framework of the DMM. The inconsistencies of using a power law and the xi stability criterion are eliminated, and a dependence with the temperature and the strain rate of the parameters involving strain rate sensitivity, efficiency and stability is naturally introduced.

(2) The new proposed model and the new rho stability criterion are able to assess and predict the behavior of any material identifying areas that are safe for hot forming and other less stable and more insecure (unsafe) zones.

(3) Our approach, and that of Prasad and Kumar, is based on the same basic stability criterion of Ziegler. However, we utilize the Garofalo equation in the entire range of the variables with continuous variations of the stability lines. This procedure avoids the formation of anomalous structures in the form of islands or complex structures in the maps in contradiction to the bases of the mechanics of continuous media.

(4) Our stability maps give zones of high and low stability, bands of high stability and a line of maximum stability in the space $\{T, \dot{\varepsilon}\}$, which agree well with the atomistic analysis of Raj. Furthermore, the influence of the forming variables on the stability can be assessed as a function of strain.

(5) While the efficiencies in the literature are evaluated in a dimensionless parameter, percent or per unit, the stability parameter that we define as rho is quantified in the dimension energy/volume or energy density in volume $\left(\mathrm{Joules} / \mathrm{m}^{3}\right)$. This allows us to assess the stability intensities, evaluating the maximum and minimum values of the parameter rho on the map and the ease of taking out the material from the stability.

(6) Applying this new rho stability criterion to a Ti-10V-4.5Fe-1.5Al alloy studied by Balasubrahmanyam and Prasad, the optimum thermomechanical conditions for hot working can be determined. Specifically, at $\varepsilon=0.2$, for $1 \mathrm{~s}^{-1}$ the model predicts an optimum stability temperature of $775{ }^{\circ} \mathrm{C}(1048 \mathrm{~K})$.

\section{ACKNOWLEDGMENTS}

The authors acknowledge the financial support of CICYT, Spain, under Program MAT2015-68919 (MINECO/FEDER).

\section{APPENDIX}

Stability Expression of Prasad from the Concepts of the DMM and the Use of a Power Law Equation

Using the power law equation:

$$
\dot{\varepsilon}=A_{\mathrm{PL}} \cdot e^{-\frac{Q_{P L}}{R \cdot T}} \cdot \sigma^{n_{P L}}
$$

and the concepts of the DMM, Prasad et al. obtained the following equations (where the subindex PL indicates that it has been obtained by adjusting the data to a power law constitutive equation):

$$
\begin{gathered}
G=\frac{P \cdot n_{\mathrm{PL}}}{n_{\mathrm{PL}}+1}=\frac{P}{m_{\mathrm{PL}}+1} \\
J=P \cdot \frac{m_{\mathrm{PL}}}{m_{\mathrm{PL}}+1}=\frac{P}{n_{\mathrm{PL}}+1}
\end{gathered}
$$

Here, $\left\{A_{\mathrm{PL}}, Q_{\mathrm{PL}}, n_{\mathrm{PL}}\right\}$ are constant values (parameters) in the equation, for a given value of strain. The fit of Eq. [A1] to the experimental set of data values $\{\dot{\varepsilon}, \sigma, T\} \quad(N$ values of the mentioned sets of three variables) results in obtaining values of the constants for a given value of strain.

The maximum value of $J$ is $J_{\max }=\frac{P}{2}$, which is reached for $m=1$. Furthermore, for this value $J_{\max }=G_{\min }=\frac{P}{2}$. This has two consequences. It is valid strictly when the power law is justified, which is in short ranges of the thermodynamic variables of the system. It is worth noting that it would be recommendable to use the total power $P$ as the denominator for the normalization of the efficiency instead of $J_{\max }$. This would make the efficiencies of $J$ and $G$ comparable and would rationally normalize the concept of efficiency. In addition, this would allow distinguishing the power used in changing the form from that in modifying the microstructure.

According to the DMM, the efficiency $\eta$ is an important parameter that synthesizes the information of the dissipative co-content and the relations of the plastic creep with the evolution of the microstructure. It is defined as:

$$
\eta_{J / J_{\max }}=\frac{J}{J_{\max }}
$$

Therefore, the efficiency of the dissipative co-content referred to its maximum performance using the values of $\mathrm{m}$ obtained from the power law is the following:

$$
\eta_{J / J_{\max }}^{\mathrm{PL}}=\frac{P \cdot \frac{m_{\mathrm{PL}}}{m_{\mathrm{L}}+1}}{\frac{P}{2}}=\frac{2 \cdot m_{\mathrm{PL}}}{m_{\mathrm{PL}}+1}
$$

Continuing with the application of the power law in the framework of DMM, from the stability criterion in its simpler form it is obtained that:

$$
\frac{\partial \operatorname{Ln}(J)}{\partial \operatorname{Ln}(\dot{\varepsilon})}>1
$$

and using $J$ obtained from the power law:

$$
J=\frac{m_{\mathrm{PL}}}{m_{\mathrm{PL}}+1} \cdot \sigma \cdot \dot{\varepsilon}
$$


All these expression from Eqs. [A1] to [A7] are given in References 33, 34 and many other works.

It is easy to see that $\ln (J)=\ln \left(\frac{m}{m+1}\right)+\ln (\sigma)+\ln (\dot{\varepsilon})$ and with the calculation of derivative operations of Eq. [16], it is obtained directly that $\frac{\left.\partial\left(\ln \frac{m}{m+1}\right)\right)}{\partial \ln (\dot{\varepsilon})}+\frac{\partial \ln (\sigma)}{\partial \ln (\dot{\varepsilon})}+\frac{\partial \ln (\dot{\varepsilon})}{\partial \ln (\dot{\varepsilon})}<1$; therefore, it is obvious that $\frac{\partial\left(\ln \left(\frac{m}{m+1}\right)\right)}{\partial \ln (\dot{\varepsilon})}+\frac{\partial \ln (\sigma)}{\partial \ln (\dot{\varepsilon})}<0$. Now, using a power law, where $m$ $=\frac{\partial \ln (\sigma)}{\partial \ln (\dot{\varepsilon})}$, the stability expression, named the Prasad or xi equation, which is well known to all researchers in this field, is finally obtained:

$$
\xi(\dot{\varepsilon})=\frac{\partial\left(\ln \left(\frac{m}{m+1}\right)\right)}{\partial \ln (\dot{\varepsilon})}+m<0
$$

\section{Algorithms Used for Determination of the Stability and Efficiency Equations}

The determination of the dissipator co-content, $J$, was carried out, as mentioned in the text, using the equation $J=\int_{0}^{\sigma} \dot{\varepsilon} \cdot d \sigma$ and the variable change $x=\alpha \cdot \sigma$. This value of $J$ with this variable change, or algorithm, was named $J(1)$. Notably, the generalized Leibniz rule was applied to reach these expressions since it was necessary to derive inside the integrals. If a transformation from the variable $\sigma$ to the variable $\dot{\varepsilon}$ is chosen, and the expression $\alpha \cdot \sigma=\sinh ^{-1}\left(\theta(\dot{\varepsilon}, T)^{G}\right)$ where $\left(\theta(\dot{\varepsilon}, T)^{G}\right)=$ $\left(\frac{\dot{\varepsilon} \cdot e^{\frac{Q^{G}}{R} T}}{A^{G}}\right)^{\left(1 / n^{G}\right)}$ is used, the following expression $J / \dot{\varepsilon}$ is obtained:

$$
J / \dot{\varepsilon}=\left(\frac{1}{\alpha^{G} \cdot\left(\theta(\dot{\varepsilon}, T)^{G}\right)^{n^{G}}}\right) \cdot \int_{0}^{\sinh ^{-1}\left(\theta(\dot{\varepsilon}, T)^{G}\right)}[\sinh (x)]^{n^{G}} \cdot \mathrm{d} x
$$

being

$$
J=\left(\frac{A^{G} \cdot e^{-\frac{Q^{G}}{R \cdot T}}}{\alpha^{G}}\right) \int_{0}^{\sinh ^{-1}\left(\theta(\dot{\varepsilon}, T)^{G}\right)}[\sinh (x)]^{n^{G}} \cdot \mathrm{d} x
$$

From this expression, substituting directly in the definition of efficiency, Eq. [17] in the text, we obtain:

$$
\eta^{G}=\frac{2 \cdot \int_{0}^{\sinh ^{-1}\left(\theta(\dot{\varepsilon}, T)^{G}\right)}[\sinh (x)]^{n^{G}} \cdot \mathrm{d} x}{\left(\theta(\dot{\varepsilon}, T)^{G}\right)^{n^{G}} \cdot \sinh ^{-1}\left[\theta(\dot{\varepsilon}, T)^{G}\right]} .
$$

The application of the Leibniz generalized rule is needed to obtain the expression of $d J / d \dot{\varepsilon}$ (for a given strain and temperature) as: $d J / d \dot{\varepsilon}=\dot{\varepsilon} \cdot \partial \sigma(\dot{\varepsilon}, T) / \partial \dot{\varepsilon}=$ $\left(1 /\left(n^{G} \cdot \alpha^{G}\right)\right) \cdot\left(\theta(\dot{\varepsilon}, T)^{G} / \sqrt{1+\left(\theta(\dot{\varepsilon}, T)^{G}\right)^{2}}\right) \cdot$ Substitut-

ing this expression in Eq. [9], it is finally obtained:

$$
\begin{aligned}
\rho= & \frac{1}{n^{G} \cdot \alpha^{G}} \cdot\left[\frac{\theta(\dot{\varepsilon}, T)^{G}}{\sqrt{1+\theta(\dot{\varepsilon}, T)^{G^{2}}}}-\left(\frac{n^{G}}{\left(\theta(\dot{\varepsilon}, T)^{G}\right)^{n^{G}}}\right)\right. \\
& \left.\cdot \int_{0}^{\sinh ^{-1}\left(\theta(\dot{\varepsilon}, T)^{G}\right)}[\sinh (x)]^{n^{G}} \cdot \mathrm{d} x\right]
\end{aligned}
$$

\section{Construction of Efficiency and Stability Maps}

An important methodological aspect is that related to software for the execution of the calculations that are necessary for the preparation of efficiency and stability maps. This is a non-trivial aspect due to the large size and complexity of multiple formulas with hyperbolic expressions, with direct and inverse functions, and all this combined with the calculations of derivatives and expressions. This can lead to the generation of different kinds of errors: truncation, rounding and cumulative.

For developing the algorithm of the efficiency and stability maps, we use the MATHCAD Professional 7.0. The program starts by defining the input parameters, which are the four adjustment coefficients of the Garofalo equation $\left\{A^{G}, Q^{G}, n^{G}, \alpha^{G}\right\}$. We give an example of the use of the program for an ideal material:

INPUT DATA (example for strain $\varepsilon=0.2$ ):

$q:=59527.10721895$ (effective activation energy in calories/mol K).

$n:=3.7104796$ (exponent of the hyperbolic sine function).

al: $=0.00338(\alpha=$ cofactor of true stress $)$.

$A=\exp (29.121729) a:=4.4402375810^{12}$ (coefficient $A$ or entropy factor).

The definition of the indexes is as follows. For the temperature and the strain rate, we use the index $i$ and $j$, respectively. The range goes from 0 to about 40 for the temperature (for large temperature ranges of about 300 $\mathrm{K}$ ) and from 0 to about 80 for the strain rate (for large strain rate intervals of about 5 orders of magnitude). The mesh size factor $i \times j$ should be $>3000$ in these cases to have a good resolution in the maps.

The expression in the Mathcad program to determine the interval for the strain rate is:

$$
E_{j}:=\exp \left(x+\frac{j}{z}\right)
$$

The value of $x$ corresponds to $\ln$ of the lowest value of the strain rate, or $j=0$, and $\ln$ of $j / z$ corresponds to the highest value, or $j=80$. For an interval of 0.001 to $100 \mathrm{~s}^{-1}$ and $j=0$ to 80 , this expression becomes: $E_{j}:=\exp \left(-6.9078+\frac{j}{6.9487}\right)$, since $-6.9078+80 / 6.9487$ $=4.6052=\ln 100$, being $\ln 0.001=-6.9078$. Obviously, 
we have identified $\ln \left(\dot{\varepsilon}_{j}\right)=\ln \left(E_{j}\right)=\left(x+\frac{j}{z}\right)$, and with the result in this case E0: $=0.001$ and $\mathrm{E} 80:=100.0$.

The expression to determine the interval for temperatures is:

Ti: $=y+w i$.

The value of $y$ corresponds to the lowest temperature in $K$, or $I=0$, and $w i$ for the highest, or $I=40$. For instance, for an interval of 900 to $1200 \mathrm{~K}$ and $I=0$ to 40, this expression becomes:

Ti: $=900+7.5 i$, since $900+7.540=1200$, and therefore $T_{0}=900 \mathrm{~K}$ and $T_{40}=1200 \mathrm{~K}$.

With this information it is possible to build the maps in a MATHCAD Professional 7.0. program. However, in case of any complications, and by prior request, we have made the program available for those interested in drawing these new maps. The importance of the correct determination of the Garofalo equation parameters before launching the program must be noted.

\section{REFERENCES}

1. A.M. Lyapunov, PhD. Thesis, Zapiski Imperatorskogo Khar'kovskogo Universiteta, 1892.

2. A.M. Lyapunov, The General Problem of Stability of Motion, Taylor and Francis Bristol, PA, 1992, reedited: Int. J. Control, vol. 55, Issue 3, 1992.

3. P.C. Parks: IMA J. Math. Control Inf., 1992, vol. 9, pp. 275-303.

4. H. Ziegler: Progr. Solid Mech., 1963, vol. 4, pp. 91-193.

5. I. Rieiro, M. Carsí, and O.A. Ruano: Metall. Mater. Trans. A, 2017, vol. 48A, pp. 3445-60.

6. Y.V.R.K. Prasad, H.L. Gegel, S.M. Doraivelu, J.C. Malas, J.T. Morgan, K.A. Lark, and D.R. Barker: Metall. Trans. A, 1984, vol. 15 A, pp. 1883-92.

7. H.L. Gegel, J.C. Malas, S.M. Doraivelu, and V.A. Shende: Metals Handbook, ASM, Metals Park, 1987, vol. 14, pp. 417-38.

8. H.L. Gegel, J.C. Malas, S.M. Doraivelu, J.M Alexander, and J.S. Gunasekera: Proc. Adv. Technol. Plast., vol. 11, K. Lange, ed., Springer, London, 1987, pp. 1243-1249.

9. J. Lu, Y. Song, L. Hua, K. Zheng, and D. Dai: J. Alloys Compd., 2018, vol. 767, pp. 865-69.

10. S.V.S.N. Murty and B.N. Rao: Mater. Sci. Eng. A, 1999, vol. 267, pp. $159-61$.

11. S.V.S.N. Murty and B.N. Rao: J. Mater. Proc. Technol., 2000, vol. 104, pp. 103-9.

12. S.V.S.N. Murty, B.N. Rao, and B.P. Kashyap: J. Mater. Proc. Technol., 2005, vol. 166, pp. 279-85.

13. S.V.S.N. Murty, B.N. Rao, and B.P. Kashyap: J. Mater. Proc. Technol., 2005, vol. 166, pp. 268-78.

14. I. Rieiro: PhD Thesis, Facultad de Ciencias Físicas, Universidad Complutense de Madrid, 1997.

15. F. Wang, J. Shen, Y. Zhang, and Y. Ning: Metals, 2019, vol. 9, pp. 844-61, https://doi.org/10.3390/met9080844.

16. R. Raj: Metall. Trans. A, 1981, vol. 12, pp. 1089-97.

17. R. Raj and M.F. Ashby: Metall. Mater. Trans. B, 1972, vol. 3B, pp. $1937-42$.

18. B.K. Raghunhat, R. Karthikeyan, and M. Gupta: Mater. Res., 2006, vol. 9, pp. 217-22.

19. G. Ganesan, K. Raghukandan, R. Karthikeyan, and B.C. Pai: Mater. Sci. Eng. A, 2004, vol. 369, pp. 230-35.

20. Y.V.R.K. Prasad: Indian J. Technol., 1990, vol. 28, pp. 435-51.
21. K.P. Rao, Y.V.R.K. Prasad, and K. Sivaram: Mater. Lett., 1990, vol. 10, pp. 66-70.

22. Y. Wang, G. Zhao, X. Xu, X. Chen, and C.S. Zhang: J. Alloys Compd., 2019, vol. 779, pp. 735-51.

23. P. Wan, K. Wang, H. Zou, S. Lu, and X. Li: J. Alloys Compd., 2019, vol. 777, pp. 812-20.

24. V.V. Balasubrahmanyam and Y.V.R.K. Prasad: Mater. Sci. Eng. A, 2002, vol. 336, pp. 150-58.

25. B.N. Sahoo and S.K. Panigrahi: J. Alloys Compd., 2019, vol. 776, pp. 865-82.

26. Y.V.R.K. Prasad: Metall. Trans. A, 1996, vol. 27, pp. 235-6.

27. S. Venugopal, S.L. Mannan, and Y.V.R.K. Prasad: Mater. Sci. Eng. A, 1993, vol. 160, pp. 63-9.

28. Y.V.R.K. Prasad and T. Seshacharyulu: Mater. Sci. Eng. A, 1998, vol. 243, pp. 82-88.

29. Y.V.R.K. Prasad and N. Ravichandran: Bull. Mater. Sci., 1991, vol. 14, pp. 1241-48.

30. Y.V.R.K. Prasad and S. Sasidhara: Hot Working Guide: A Compendium of Processing Maps, ASM International, Materials Park, 1997.

31. A.K.S. Kumar: MS Thesis, Bangalore, India, 1987.

32. J. Castellanos, I. Rieiro, M. El Mehtedi, M. Carsí, and O.A. Ruano: Int. J. Mater. Res., 2010, vol. 101, pp. 787-93.

33. I. Rieiro, O.A. Ruano, M. Eddahbi, and M. Carsí: J. Mater. Proc. Technol., 1998, vol. 78, pp. 177-83.

34. I. Rieiro, M. Carsí, and O.A. Ruano: Mater. Sci. Technol., 2009, vol. 25 , pp. $995-1002$.

35. I. Rieiro, V. Gutierrez, J. Castellanos, M. Carsí, M.T. Larrea, and O.A. Ruano: Metall. Mater. Trans. A, 2010, vol. 41A, pp. 2396-407.

36. V. Gutierrez, I. Rieiro, M. Carsí, and O.A. Ruano: Rev. Metal. MADRID, 2013, vol. 49, pp. 378-96.

37. Y. Wu, Y. Liu, C. Li, X. Xia, Y. Huang, H. Li, and H. Wang: $J$. Alloys Compd., 2017, vol. 712, pp. 687-95.

38. S. Zeng, A. Zhao, H. Jiang, and Y. Ren: J. Alloys Compd., 2017, vol. 698, pp. 786-93.

39. Y. Zhou, Y. Liu, X. Zhou, Ch. Liu, J. Yu, Y. Huang, H. Li, and W. Li: J. Mater. Sci. Technol., 2017, vol. 33, pp. 1448-56.

40. Y. Zhou, Y. Liu, X. Zhou, Ch. Liu, L. Yu, C. Li, and B. Ning: $J$. Mater. Res., 2015, vol. 30, pp. 2090-100.

41. Y. Xu, C. Chen, J. Jia, X. Zhang, H. Dai, and Z. Bai: Metall. Mater. Trans. B, 2018, vol. 49B, pp. 3470-87.

42. H.E. Hu, X.Y. Wang, and L. Deng: Mater. Sci. Technol., 2014, vol. 30 , pp. 1321-27.

43. F. Montheillet, J.J. Jonas, and K.W. Neale: Metall. Trans. A, 1996, vol. 27, pp. 232-35.

44. S. Ghosh: Metall. Mater. Trans. A, 2002, vol. 33A, pp. 1569-72.

45. F. Garofalo: Trans. AIME, 1963, vol. 227, pp. 351-57.

46. A. Rudra, M. Ashiq, S. Das, and R. Dasgupta: Metall. Mater. Trans. B, 2019, vol. 50B, pp. 1060-76.

47. A. Momeni, K. Dehghani, G.R. Ebrahimi, and S. Kazemi: Metall. Mater. Trans. A, 2013, vol. 44A, pp. 5567-76.

48. A. Hamada, T. Juuti, A. Khosravifard, A. Kisko, P. Karjalainen, D. Porter, and J. Kömi: Mater. Des., 2018, vol. 154, pp. 117-29.

49. K.A. Babu, S. Mandal, C.N. Athreya, B. Shakthipriya, and V.S. Sarma: Mater. Des., 2017, vol. 115, pp. 262-75.

50. S. Wang, J.R. Luo, L.G. Houa, J.S. Zhang, and L.Z. Zhuang: Mater. Des., 2017, vol. 113, pp. 27-36.

51. Z. Du, S. Jiang, and K. Zhan: Mater. Des., 2015, vol. 86, pp. 464-73.

52. C. Zhang, L. Zhang, W. Shen, C. Liu, and R. Li: Mater. Des., 2016, vol. 90, pp. 804-814.

Publisher's Note Springer Nature remains neutral with regard to jurisdictional claims in published maps and institutional affiliations. 